\title{
Does Imprecision in The Waggle Dance Fit Patterns Predicted by The Tuned-Error Hypothesis?
}

\author{
David A. Tanner • P. Kirk Visscher
}

Revised: 15 October 2009 / Accepted: 14 January 2010 /

Published online: 23 February 2010

(C) The Author(s) 2010. This article is published with open access at Springerlink.com

\begin{abstract}
The waggle dance of the honey bee is used to recruit nest mates to a resource, though direction indicated for a resource may vary greatly within a single dance. Some authors suggest that this variation exits as an adaptation to distribute recruits across a patch of flowers, and that, due to the variation's inverse relationship with distance, the shape of the recruit distribution will remain constant for resources at different distances. In this study, we test this hypothesis by examining how variation in the indication of direction and distance changes with respect to distance. We find that imprecision in the communication of direction does not diminish rapidly enough to accommodate an adaptive-error hypothesis, and we also find that variation in the indication of distance has a positive relationship with the distance of a resource from the hive.
\end{abstract}

Keywords Tuned-error/waggle $\cdot$ dance/distance $\cdot$ communication

\section{Introduction}

Evolution is the biological process primarily responsible for the rich diversity of Earth's biota and is accomplished most prominently through natural selection (Darwin 1859). The potential effect of selective pressures on any organism, and the form and function that a trait may realize, can be limited, however, by the current state and evolutionary history of that trait (Gould and Lewontin 1979). Occasionally, students of evolution "atomize" an individual into discrete and independent traits without considering how traits interrelate and influence the evolution of the entire organism. This tendency may lead to ingenious or fanciful explanations of the

D. A. Tanner (凶)

Biology Department, Utah State University, 5305 Old Main Hill, Logan, UT 84322, USA

e-mail: dtanner@biology.usu.edu

P. K. Visscher

Entomology Department, University of California, Riverside, Riverside, CA 92521, USA 
adaptive nature of a trait, and also to the misapprehension that all character states in nature are products of natural selection and exist as optima (Gould and Lewontin 1979).

Of recent interest is the utility of imprecision in the waggle dance of the honey bee. The dance is an adaptation for social recruitment (Sherman and Visscher 2002; Dornhaus and Chittka 2004; Dornhaus et al. 2006) and is used to communicate the location of that resource relative to the position of the sun (von Frisch 1967). The dance works as a ritualized reenactment of the foraging flight, in which the direction flown to a resource, relative to the sun's azimuth, is indicated by the body orientation and movement of the dancing bee relative to "up" on a vertical comb (Fig. 1). This phase of the waggle dance is called a waggle phase. After completing a waggle phase, a dancer returns to its origin via a return phase to perform another waggle phase. Distance is communicated through sound bursts produced during the waggle phase of the dance, the duration of which correlates to the distance of a resource (Wenner 1962; Esch and Kerr 1965; von Frisch 1967).

There is considerable variation, however, in the direction communicated within a single dance (Haldane and Spurway 1954; Wilson 1962; von Frisch 1967; Edrich 1975; Gould 1975; Towne 1985; Towne and Gould 1988; Weidenmüller and Seeley 1999). Though some of this error may be mitigated by the manner in which information from the dance is processed (Tanner and Visscher 2008). The angular difference in the direction between consecutive waggle phases is the divergence angle. Interestingly, the magnitude of the divergence angle has a negative relationship with the distance of a resource from the hive. Some authors contend that the divergence angle has evolved as an adaptation in temperate honey bees to distribute recruits across a resource patch (Wilson 1962; Towne and Gould 1988), and that the relationship between divergence angle and the distance of a resource from the hive represents a tuning to produce a recruit distribution of optimal size that remains constant with distance (Towne 1985; Towne and Gould 1988; Weidenmüller

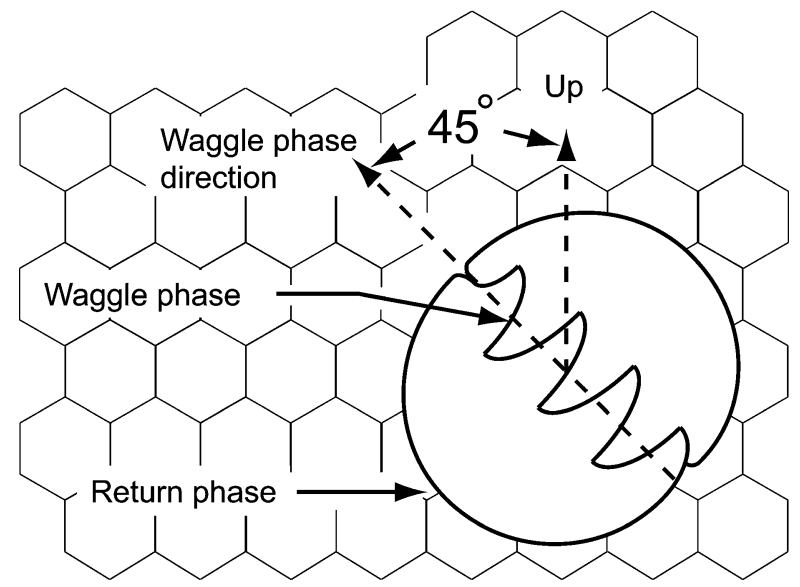

Fig. 1 Waggle dance of the honey bee. In this figure, the waggle phase is oriented $45^{\circ}$ to the left of "up" in the hive, communicating that a resource is located $45^{\circ}$ to the left of the sun's azimuth. Upon completing this waggle phase, the dancer bee will return to its origin via a return phase, and perform another waggle phase that may indicate a different direction. 
and Seeley 1999). A recent study of recruit distributions, however, showed that, at a distance of $250 \mathrm{~m}$ from the hive, the distribution of bees was significantly smaller than the imprecision in the dance would predict (Tanner and Visscher 2008). In this study, we test if the imprecision in the indication of distance and direction are sufficient to produce a distribution of recruits that will have the same spatial dimensions as a resource becomes increasingly distant. For a distribution to remain constant with distance, not only must the imprecision in direction decrease, but also the imprecision in distance communication must remain constant. An increase or decrease in variation will alter the size of the distribution.

\section{Materials and Methods}

To test what effect the distance of a resource has on the imprecision in the communication of direction and distance, and consequently on the distribution of recruits, we conducted an experiment in which bees were trained to feeders located $200,400,600,800$, and 1,000 $\mathrm{m}$ from the hive. We recorded the dances and dance sounds of these bees and compared the precision in these dances to models of dance error generated by the tuned-error hypothesis.

\section{Bees and Observation Hive}

We used two colonies of bees of mixed European lineage in this experiment; each colony consisted of approximately 2,000 bees. We housed the colonies in observation hives consisting of 2 vertically stacked frames of mixed honey and brood, and wire mesh walls ( $3 \mathrm{~mm}$ mesh size). In order to prevent light from reaching the surface of the hive, which may affect the precision of dancing (Tanner 2003), we placed the observation hives in a darkened, windowless building. Bees entered the hive through a short length of PVC tube that extended from the hive and through the wall of the building. We fitted the interior end of the tube with a wooden wedge so that all bees were directed to the side of the observation hive facing the video camera.

The observation hive and building were located at the Agricultural Experiment Station of the University of California, Riverside amid citrus and Eucalyptus trees, small herbaceous plots of cultivated vegetables, and open fields. There was very little ambient forage available, however, when this experiment was performed.

\section{Training and Data Collection}

In this experiment, we trained bees from each colony to feeders located at various distances from the hive by placing a feeder of rich sucrose $(2 \mathrm{M})$ scented with peppermint oil $(15 \mu \mathrm{L} / \mathrm{L})$ directly in front of the colony entrance. Once bees were actively feeding, we moved the feeder $200 \mathrm{~m}$ from the hive in small increments. Each bee that arrived at the feeder received a plastic numbered tag, and the dances of these marked bees were video recorded. We included dances consisting of at least 20 consecutive waggle phases in our analysis of dance error.

Dances, illuminated in infrared light, were recorded with a Sony handycam DCRHC20 digital camcorder on a tripod $0.5 \mathrm{~m}$ from the hive. We recorded dance sounds 
to the audio track of the digital video tape with a Radio Shack condenser microphone element, first passing the signal through a 15 band graphic equalizer that selectively amplified sounds between 200 and 1,000 Hz. Each bee that successfully performed a dance was collected in a small plastic bag, and placed in a cooler. Once dances from 16 bees were collected, we moved the feeder in small increments $400,600,800$, and then $1,000 \mathrm{~m}$ from the hive repeating the same procedure at each distance.

\section{Video Transcription and Data Analysis}

We reviewed the video of dancing bees in 1/6 real time speed using iMovie software, and measured the direction of each waggle phase by tracing the path of the vertex of the dancing bee's head onto a sheet of acetate suspended against a flat screen computer monitor. Next, using a computer program (written by P. K. Visscher U.C. Riverside) that records the $\mathrm{x}, \mathrm{y}$ coordinates of the pixel at the start and end point of each traced waggle phase, we calculated the angle, relative to "up" in the hive, and the length of each waggle phase. We define the start point of the waggle phase as the point at which the bee began waggling, and the end point as either the point at which waggling stopped, or the point at which the bee began to exit from the waggle phase to the return circuit. Divergence angle was then calculated as the angular difference between consecutive waggle phases, and we calculated the mean divergence angle for each bee in each treatment at each distance. We also calculated the difference between the direction indicated during a waggle phase following a left or right turn and the true direction to the resource. We then calculated a mean "left divergence" and "right divergence", as well as the standard error of the mean, mode, and the range (Table 1 and Fig. 2). We also modeled a divergence angle-to-distance relationship that would result in arcs (i.e. predicted angular distribution of recruits in the field) subtended by the divergence angles of equal size at all distances, and

Table 1 Mean Divergence Angle and Error in Left and Right Waggle Phases

\section{Dance Error}

\begin{tabular}{llllll}
\hline & $200 \mathrm{~m}$ & $400 \mathrm{~m}$ & $600 \mathrm{~m}$ & $800 \mathrm{~m}$ & $1,000 \mathrm{~m}$ \\
\hline $\begin{array}{llll}\text { Mean DA } \\
\text { Left WP }\end{array}$ & 28.67 & 32.97 & 22.24 & 12.79 & 10.05 \\
Mean difference & 15.56 & 12.85 & 6.32 & 8.76 & 6.17 \\
Standard Err & 0.53 & 0.57 & 0.37 & 0.43 & 0.33 \\
Mode & 13 & 10 & 1 & 1 & 1 \\
Range & 43 & 37 & 43 & 29 & 30 \\
Right WP & 14.9 & 11.67 & 6.57 & 8.33 & 6.51 \\
Mean difference & 0.54 & 0.49 & 0.36 & 0.44 & 0.36 \\
Standard Err & 13 & 14 & 1 & 2 & 3 \\
Mode & 50 & 34 & 41 & 36 & 33 \\
Range & & & & & \\
\end{tabular}




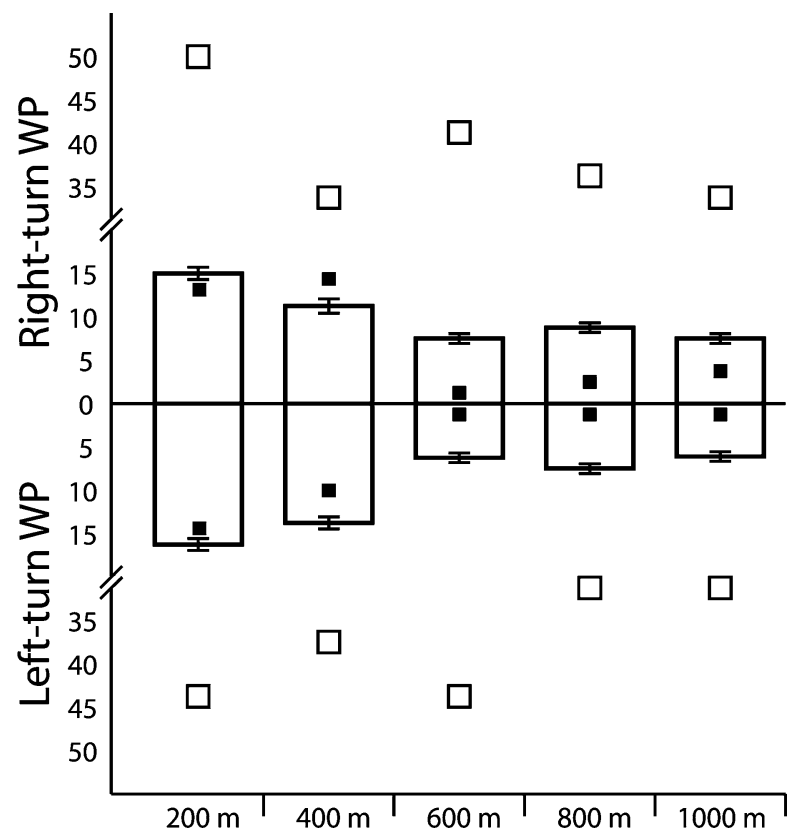

Fig. 2 Analysis of variation in the dance of the honey bee. The bars represent the mean difference between the direction indicated in a waggle phase succeeding a right turn (top) or left turn (bottom) at a given distance. The error bars represent the standard error of the mean, the closed squares represent the mode, and the open squares represent the range, or greatest difference.

compared it with the observed relationship (Fig. 3). The tuned-error hypothesis predicts that the change in divergence angle, or rather the predicted distributions of bees generated from a change in the divergence angle, should track one of the isoarc-lengths (gray lines).

We quantified the duration of the dancing bees' sound bursts using Raven Lite 1.0 software by measuring the elapsed time between the beginning and end of sound production within a waggle phase. To reduce the background noise, we used the Raven Lite filter to dampen noise that was less than $200 \mathrm{~Hz}$ and greater than $1,000 \mathrm{~Hz}$. We then generated an oscillograph for each waggle phase, which we used to measure the duration of each sound burst. To test for an effect of the distance of a resource on the duration of sound bursts, we calculated the mean duration of the sound bursts at each distance, and performed an Analysis of Variance using mean burst duration as the dependent variable, and distance of the resource from the hive as the independent variable. To test for an effect of resource distance on variation in burst duration, we performed a regression analysis in which we regressed the variance in sound burst duration against the distance of the resource from the hive.

\section{Results}

Figure 3 depicts the divergence angle to distance relationship that would result in arcs (i.e. the lateral distributions of bees) of equal width at all distances, as a family 


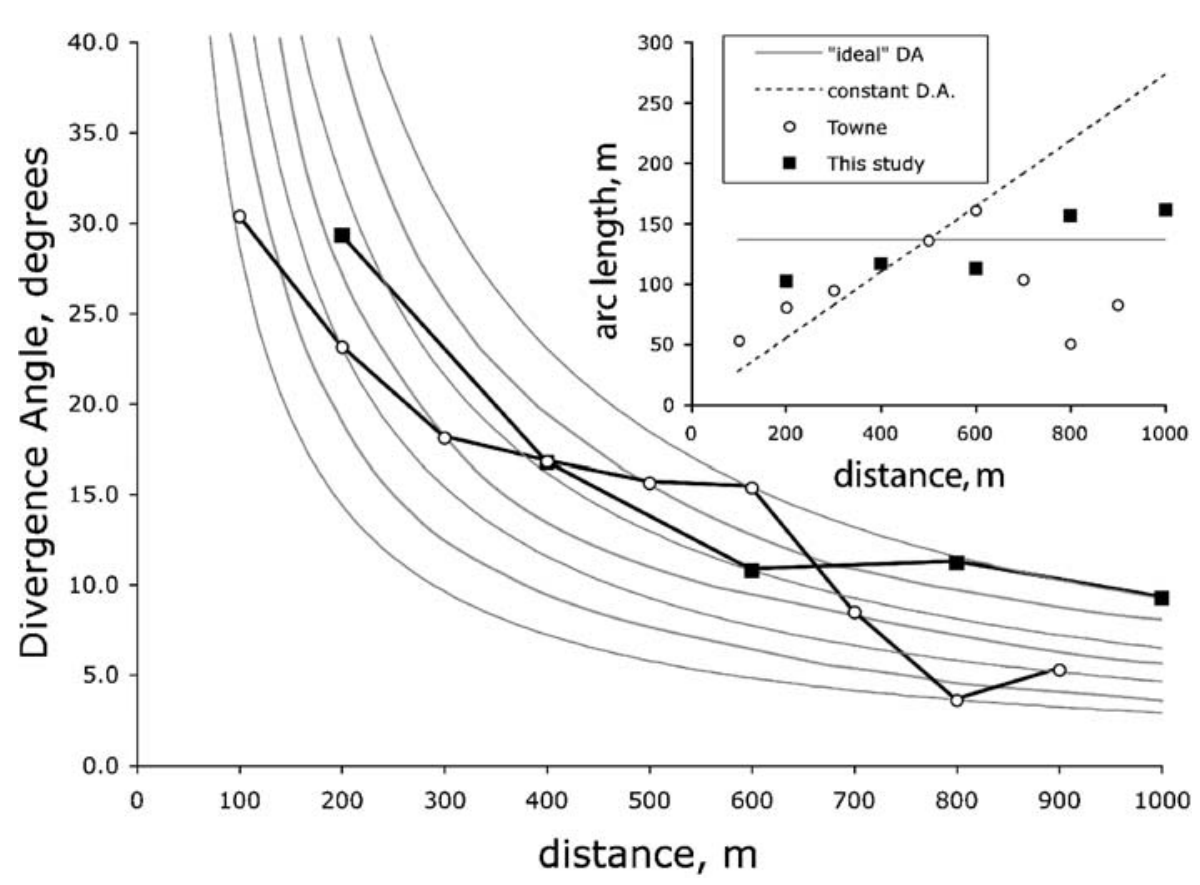

Fig. 3 Decrease in divergence angle with distance graphed against predicted pattern of decrease. Open circles are data taken from Towne and Gould (1988), black squares represent data that we collected.

of iso-arc-length lines (gray lines). The black lines and points represent the data we collected and previously published data of Towne and Gould (1988). The tuned-error hypothesis states that distributions of recruits, resulting from the imprecision in the dance, will have spatial dimensions that are constant despite the distance of a resource. If the imprecision in the dance is sufficient, then the data illustrated on Fig. 3 should follow a single iso-arc-length line. We show, however, that the distributions of bees (black lines), as predicted by dance information, do not track the iso-arc-lengths (gray lines).

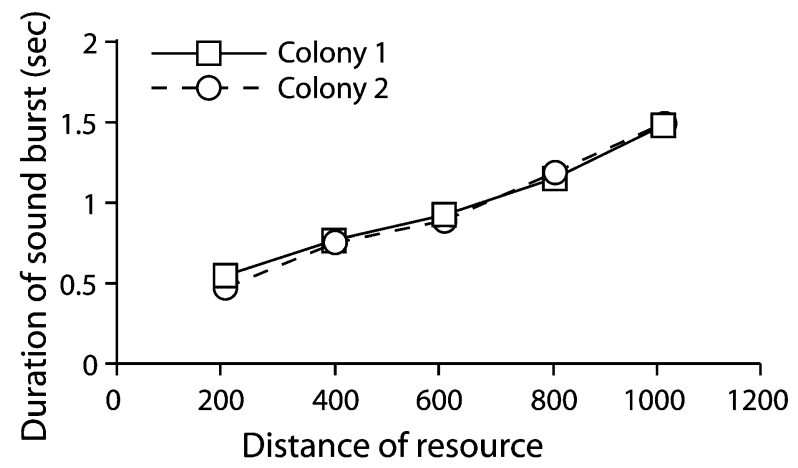

Fig. 4 The effect of resource distance on the duration of sound bursts. 
Table 2 Analysis of Variance for the Effects of Distance on Divergence Angle

\begin{tabular}{lccc}
\hline Source & DF & F & $P$ \\
\hline Distance & 4 & 158.9 & $<0.0001$ \\
Colony & 1 & 0.00 & 0.9503 \\
Colony*Distance & 4 & 2.78 & 0.0282 \\
\hline
\end{tabular}

The graph in the upper right of Fig. 3 plots an arc length (i.e. lateral distribution of bees) to distance relationship produced by holding divergence angle constant at the observed $500 \mathrm{~m}$ value (slanted dashed lines), and holding arc length constant with distance (horizontal light line), which represents the relationship predicted by the tuned-error hypothesis. Neither our, nor the data reported by Towne and Gould (1988), fit the relationship predicted by the tuned-error hypothesis of constant arc length with distance. Instead, there are increasing arc lengths (i.e. increasing area over which recruits will be distributed) up to about $500 \mathrm{~m}$, and then steady or decreasing arc lengths.

Figure 4 shows the relationship between sound burst duration and the distance of a resource, and Table 2 contains the results of the ANOVA. These results show that the distance of a resource has a significant effect on sound duration $(F=158.9, P<$ 0.0001 ), while all other effects were not significant.

The regression of variance in the dance against the distance of the resource from the hive was significant $\left(r^{2}=0.345, P<0.001\right)$, which is consistent with data presented in von Frisch (1967). Figure 5 shows that the variance of sound duration increased with increasing distance.

\section{Discussion}

We show that imprecision in dance communication does not fit specific trends predicted by the tuned-error hypothesis. While divergence angle in dances decreases as the distance of a resource increases, it does not decrease rapidly enough to create

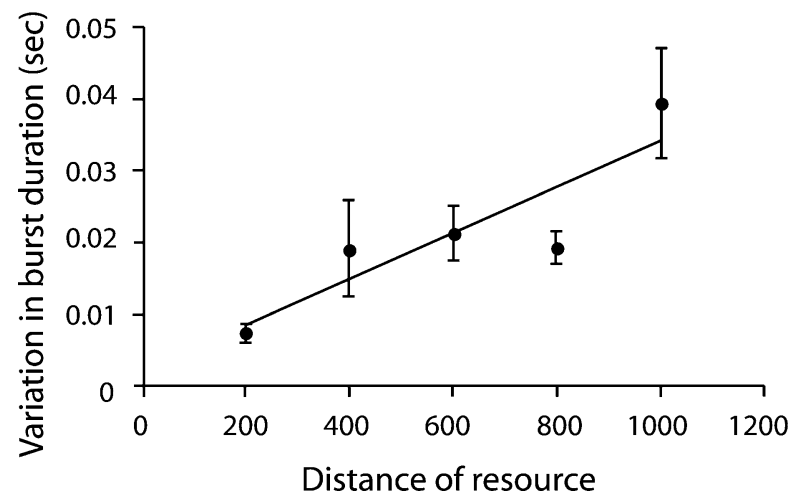

Fig. 5 The effect of resource distance on the variation in sound burst duration. 
a spatial distribution of recruits that will remain constant (Fig. 3). Indeed, when resources are located within $500 \mathrm{~m}$ from the hive, a model of no change in divergence angle with distance fits the pattern of recruit distribution, as predicted by dance information, better than a model of the tuned-error hypothesis, suggesting that distributions of recruits would increase at a steady rate despite smaller divergence angles. Additionally, we show that the variation in distance communication increases significantly with distance, which may increase the distribution of recruits in the field. We note, however, that while this change in variation is significant, it is not large (Fig. 5). These data suggest that the error in the dance would produce distributions of recruits that noticeably increase in size with the distance of a resource from the hive. This trend was illustrated previously by Towne (1985), who showed a distribution of bees covering an area of $9,920 \mathrm{~m}^{2}$ at a distance of $100 \mathrm{~m}$ from the hive and $20,120 \mathrm{~m}^{2}$ at $800 \mathrm{~m}$ from the hive, though these data were reported to support the tuned-error hypothesis by illustrating distributions of similar sizes at various distances from the hive.

In this study, we tested predicted distributions, and not actual distributions, of recruits against patterns predicted by the tuned-error hypothesis, though our data corroborate previous studies that show that recruit distributions do not fit specific patterns predicted by the tuned-error hypothesis (Towne 1985; Tanner and Visscher 2008). We show here that the imprecision in the communication of direction does not decrease rapidly enough and imprecision in the communication of distance increases too much to generate patterns predicted by the tuned-error hypothesis. We suggest, therefore, that natural selection need not be invoked to explain imprecision in the honey bee dance language, but that it may be more simply explained as an adaptive constraint, and echo the remarks of Williams (1966) on natural selection:

"This biological principle should be used only as a last resort. It should not be invoked when less onerous principles, such as those of physics and chemistry

... are sufficient for a complete explanation."

Acknowledgements We would like to acknowledge and thank Dr. Richard Redak, and Dr. John Klotz for their thoughtful review of this manuscript. We would also like to thank the Regents of the University of California and Graduate Student Association of UC Riverside for funding.

Open Access This article is distributed under the terms of the Creative Commons Attribution Noncommercial License which permits any noncommercial use, distribution, and reproduction in any medium, provided the original author(s) and source are credited.

\section{References}

Darwin CR (1959) On the origin of species. Random House, New York

Dornhaus A, Chittka L (2004) Why do honey bees dance? Behav Ecol Sociobiol 55:395-401

Dornhaus A, Klügl F, Oechslein C, Puppe F, Chittka L (2006) Benefits of recruitment in honey bees: effects of ecology and colony size in an individual-based model. Behav Ecol 17:336-344

Edrich W (1975) The waggle dance of the honey bee: a new formulation. Fortschr Zool 23:20-30

Esch H, Kerr WE (1965) Sound: an element common to communication of stingless bees and to dances of the honey bee. Science 149:320-321

Gould JL (1975) Honey bee recruitment: the dance-language controversy. Nature 252:300-301 
Gould SJ, Lewontin RC (1979) The spandrels of San Marco and the Panglossian paradigm: a critique of the adaptationist programme. Proc R Soc Lond B Biol Sci 205(1161):581-598

Haldane JBS, Spurway H (1954) A statistical analysis of communication in Apis mellifera and a comparison with communication in other animals. Insectes Soc 1:247-283

Sherman G, Visscher PK (2002) Honey bee colonies achieve fitness through dancing. Nature 419:920922

Tanner DA (2003) An evaluation of the tuned-error hypothesis in the honey bee. MS thesis, University of California - Riverside

Tanner DA, Visscher PK (2008) Do honey bees average directions in the waggle dance to determine a flight direction? Behav Ecol Sociobiol 62(12):1891-1898

Towne W (1985) The spatial precision and mechanisms of the dance communication of honey bees: experimental and comparative studies. PhD thesis, Princeton University Press, Princeton, NJ

Towne W, Gould J (1988) The spatial precision of the honey bees' dance communication. J Insect Behav 1:129-155

von Frisch K (1967) The dance language and orientation of bees. Harvard University Press, Cambridge

Weidenmüller A, Seeley T (1999) Imprecision in the waggle dances of the honeybee (Apis mellifera) for nearby food sources: error or adaptation? Behav Ecol Sociobiol 46:190-199

Wenner AM (1962) Sound production during the waggle dance of the honey bee. Anim Behav 10:79-95

Williams GC (1966) Adaptation and natural selection. Princeton University Press, Princeton

Wilson EO (1962) Chemical communication among workers of the fire ant Solenopsis saevissima (Fr. Smith). Anim Behav 10:148-158 